\title{
Labyrinthe
}

\section{Le système de l'Encyclopédie et la métaphore de la lumière : héritage et refonte du système cartésien}

\section{Marion Chottin}

\section{Q OpenEdition}

12 Journals

Édition électronique

URL : http://journals.openedition.org/labyrinthe/4054

DOI : $10.4000 /$ labyrinthe.4054

ISSN : 1950-6031

Éditeur

Hermann

Édition imprimée

Date de publication : 1 février 2010

Pagination : $37-49$

ISBN : 978-2-7056-6984-3

\section{Référence électronique}

Marion Chottin, «Le système de l'Encyclopédie et la métaphore de la lumière : héritage et refonte du système cartésien », Labyrinthe [En ligne], 34 | 2010 (1), mis en ligne le 01 février 2012, consulté le 01 mai 2019. URL : http://journals.openedition.org/labyrinthe/4054 ; DOI : 10.4000/labyrinthe.4054 


\title{
Le système de l'Encyclopédie et la métaphore de la lumière : héritage et refonte du système cartésien
}

\author{
Marion CHOTTIN \\ mchottin@yahoo.fr
}

La philosophie post-moderne, en particulier sous les plumes de Horkheimer et Adorno, a mis en cause la capacité, pour un système du savoir, de pouvoir constituer autre chose que l'expression d'une raison 《 totalitaire $»^{1}$ et « mythologique $»^{2}$, impuissante à épancher sa soif illusoire d'embrasser la totalité du réel. Cependant, tout savoir qui se met en système se clôture-t-il par son désir de s'emparer de l'Univers par la pensée, ou bien un tel ordonnancement des connaissances peut-il demeurer ouvert, pour constituer celui d'une pensée libre et consciente de ses limites? Dans cette optique, la façon dont, au XVIII ${ }^{\mathrm{e}}$ siècle, les Encyclopédistes ont pensé leur entreprise de systématisation du savoir dans une relation critique à Descartes apparaît comme un moyen particulièrement fécond d'évaluer les thèses de Horkheimer et Adorno. Car au fondement $\mathrm{du}$ 《système figuré des connaissances humaines ${ }^{3} » \mathrm{de}$ l'Encyclopédie se trouve ce par quoi les sciences peuvent être connues, à savoir l'entendement humain. Or, Diderot et d'Alembert reconduisent par là le geste inaugural de Descartes qui, dans les Règles pour la direction de l'esprit ${ }^{4}$, a considéré l'esprit humain comme le pôle unificateur de toutes les sciences. De surcroît, les Encyclopédistes justifient le rôle

1. Max Horkheimer et Theodor Adorno, La Dialectique de la Raison, Paris, Gallimard, coll. « Tel », [1944] 1974, p. 24.

2. Si cet adjectif n'est pas attribué tel quel à la «Raison », il n'en demeure pas moins que toute la première partie du chapitre « Le concept d'Aufklärung » est consacrée à la démonstration de l'intrication du mythe et de la raison, celui-là étant toujours déjà du rationnel, et celle-ci de la mythologie déguisée.

3. Jean Le Rond d'Alembert, Discours préliminaire de l'Encyclopédie, Paris, Vrin, [1751] 2000, p. 180. Ce texte, de nature programmatique en ce qu'il annonce les différents ordres que devra suivre l'Encyclopédie, est suivi du «système figuré des connaissances humaines ». Ce dernier dispose sous la forme de l'arbre l'un des ordres dont les principes sont exposés dans le Discours, l'ordre encyclopédique d'exposition des connaissances. Dans la suite de l'article, nous désignons le Discours avec le sigle $D P$. 4. Descartes fut en cela précédé de Francis Bacon, Du Progrès et de la promotion des savoirs, avantpropos, traduction et notes par Michèle Le Doeuff, Paris, Gallimard, coll. « Tel », 1991. Sur les rapports 
ainsi dévolu à l'entendement en usant de la même métaphore que celle à laquelle Descartes avait recourue en 1627 : pour Diderot et d'Alembert aussi bien que pour l'auteur des Regulae, l'esprit humain est au fondement du système parce qu'il détient une lumière dont le faisceau peut rassembler en une unité la diversité des sciences. Aussi la métaphore du soleil, dont Derrida souligne pourtant l'essentielle imperfection ${ }^{5}$, donne à penser rien de moins que la systématicité des connaissances. Mais, d'après les Encyclopédistes, ce trope conditionne chez Descartes un ordonnancement des sciences et de leurs objets qui ne présente ni faille ni lacune, et surpasse ainsi les limites inhérentes à l'entendement humain. Comment peuvent-ils donc en même temps donner à leur édifice théorique la même fondation que celle sur laquelle Descartes a bâti son système, et refuser la prétention d'appréhension totale de l'Univers qu'une telle fondation paraît justement conditionner? Les Encyclopédistes sont-ils parvenus sur ce terrain à construire un système différent de celui qu'ils attribuent à Descartes? Ou la reprise de la métaphore cartésienne les a-t-elle contraint à élever un système équivalent à celui qu'ils ont voulu détruire?

\section{Un héritage en demi-teinte}

Dans les Regulae, les sciences ne sont plus distinguées, comme chez Aristote $^{6}$, en fonction des genres d'objets auxquels elles s'appliquent, mais unifiées par la lumière du sujet, qui demeure la même quel que soit

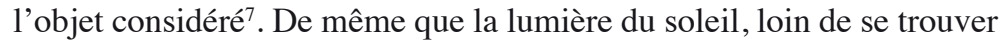

qu'entretient l'arbre encyclopédique avec l'arbre baconien, voir Michel Malherbe, « Bacon, Diderot et l'ordre encyclopédique », Revue de synthèse, (1) 1994, p. 13-37.

5. Marges de la philosophie, Paris, Les Éditions de Minuit, 1972, « La mythologie blanche. La métaphore dans le texte philosophique », p. 299.

6. Métaphysique, Paris, Vrin, traduction Jules Tricot, 1991, livre T, chap. II, 1004 a, p. 114. Pour le Stagirite, la philosophie première (ou théologie) occupe dans la classification des sciences la place éminente en tant qu'elle traite des êtres à la fois immobiles et « séparés », et précède en cela les mathématiques qui s'occupent des êtres immobiles mais non «séparés », qui elles-mêmes précèdent la physique, occupée des êtres « séparés » mais mobiles. Aussi l'ordonnancement des sciences dépend-il alors du genre d'êtres que celles-ci prennent en considération, genre qui les maintient dans des sphères parfaitement étanches, selon un principe de subordination fondé sur la dignité ontologique de leurs objets.

7. «Toutes les sciences n'étant rien d'autre que la sagesse humaine, qui reste toujours une et la même, quelque soit la différence des sujets auxquels on l'applique, et qui ne leur emprunte pas plus de distinctions que la lumière du soleil n'en emprunte à la variété des choses qu'il éclaire, il n'est besoin 


\section{Système des connaissances et système du monde}

modifiée par les objets qui la réfléchissent, les enveloppe tous d'une même luminosité, la lumière de l'esprit humain, toujours une et semblable à elle-même, unifie la diversité des objets auxquels elle s'applique ${ }^{8}$. Les sciences, loin d'être séparées en vertu des différents genres d'êtres qu'elles considèrent, s'enchaînent en tant que l'évidence d'un objet rend possible la connaissance du suivant. La lumière du sujet vient les lier en même temps qu'elle enchaîne les uns aux autres leurs objets respectifs.

C'est donc en héritier de Descartes que D'Alembert, dans le $D P$, attribue au sujet de connaissance le statut de fondement du système encyclopédique:

Ce sont des branches [sc. les différentes sciences de l'ordre encyclopédique] qui partent d'un même tronc, savoir de l'entendement humain'.

De la même façon que leur prédécesseur, les Encyclopédistes ${ }^{10}$ considèrent les sciences liées entre elles, et de surcroît unifiées par le sujet qui préside à leur liaison.

L'héritage de Descartes se remarque jusque dans la façon dont les Encyclopédistes justifient la place de fondement du système qu'ils attribuent à l'entendement humain. Sur ce point, c'est sous la plume de Diderot que la proximité des philosophes des Lumières avec l'auteur des Regulae se signale le plus nettement:

Une considération surtout qu'il ne faut point perdre de vue, c'est que si l'on bannit l'homme ou l'être pensant et contemplateur de dessus la surface de la terre; ce spectacle pathétique et sublime de la nature n'est plus qu'une scène triste et muette. L'univers se tait; le silence et la nuit s'en emparent. Tout se change en une vaste solitude où les phénomènes inobservés se passent d'une manière obscure et sourde. [...] Pourquoi n'introduironsnous pas l'homme dans notre ouvrage, comme il est placé dans l'univers? Pourquoi n'en ferons-nous pas un centre commun? [...] Voilà ce qui nous

d'imposer aux esprits aucune limite », René Descartes, Règles pour la direction de l'esprit, Paris, Vrin, [1627] 1996, traduction et notes par Jean Sirven, règle 1, p. 2.

8. Jean-Luc Marion, Sur l'ontologie grise de Descartes, Paris, Vrin, [1975] 1993, p. 29.

9. Op. cit., p. 71.

10. Il s'agit également de la thèse de Diderot, qui, dès la rédaction du Prospectus en 1750, a initié le travail d'élucidation conceptuelle du système encyclopédique. 


\section{Labyrinthe, $n^{\circ} 34$}

a déterminé à chercher dans les facultés principales de l'homme, la division générale à laquelle nous avons subordonné notre travail ${ }^{11}$.

De même que dans les Regulae, le sujet connaissant préside à l'ordonnancement des sciences en vertu de sa capacité à éclairer chacun des objets à connaître, l'Encyclopédie fait de l'homme son ultime fondement en raison de la lumière que sa position centrale dans l'Univers lui fait répandre sur toute chose. Mais ce n'est pas à dire que Diderot, dans le sillage de Descartes, attribue à l'esprit humain le rôle du soleil qui, chez Platon $^{12}$, conditionnait aussi la connaissance, mais qui, loin d'être le symbole du sujet connaissant, incarnait au contraire ce vers quoi l'homme devait tourner ses regards, à savoir l'Idée du Bien.

Selon Diderot, si l'homme occupe le centre de l'Univers, c'est qu'il constitue la fin de la connaissance ${ }^{13}$ : le savoir tout entier doit servir à son bonheur, c'est pourquoi toutes les choses lui seront reliées comme à un centre commun. Une conception pragmatiste de la connaissance, fondée sur un hédonisme revendiqué, justifie donc pour Diderot la centralité de l'homme au sein de l'Univers, et, par là même, son statut de fondement du système encyclopédique.

Or, s'il est encore une fois visible, l'héritage cartésien sur la pensée des Lumières commence ici à devenir plus ambigu. Certes, l'auteur des Regulae subordonne la connaissance au bien-être de l'homme ${ }^{14}$; et pour Diderot, l'ego est posé de façon a priori au fondement du système en tant qu'il en constitue la fin pratique: puisque l'ordonnancement des sciences doit servir les intérêts humains, la construction de l'arbre devra partir de l'homme, et envisager de là comment les différentes sciences peuvent lui être rapportées. Mais, si, chez Descartes, les sciences doivent bien aider les hommes à vivre mieux, elles ne résultent pas pour lui, comme pour les

11. Article «Encyclopédie », Encyclopédie, ou Dictionnaire raisonné des sciences, des arts et des métiers par une société de gens de lettres, en 17 tomes, Paris, chez Briasson, David l'aîné, Le Breton, Durand, 1751-1765, t. v (1755), p. 641 a-b.

12. La République, Paris, Flammarion, traduction inédite, introduction et notes par Georges Leroux, Paris, coll. GF, 2002, livre vi, 508a-509c.

13. Cf. Denis Diderot, Article «Encyclopédie», op. cit., p. 641 b: «L'homme est le terme unique d'où il faut partir, et auquel il faut tout ramener, si l'on veut plaire, intéresser, toucher, jusque dans les considérations les plus arides et les détails les plus secs. Abstraction faite de mon existence et du bonheur de mes semblables, que m'importe le reste de la nature? ». Souligné par nous.

14. Op. cit., p. 4. 


\section{Système des connaissances et système du monde}

Encyclopédistes, de la recherche du bien-être matériel, mais davantage d'un désir de connaître qui ne forme pas moins la raison de leur utilité future.

Par conséquent, le système encyclopédique paraît faire bien autre chose que de parachever ${ }^{15}$ le système cartésien: substituer à la lumière naturelle de Descartes, qui confère un intérêt à ce qu'elle éclaire du fait même qu'elle l'éclaire, une lumière qui se projette sur ce qui présente pour elle un intérêt - même s'il s'agit pareillement de l'Univers tout entier. Tandis que chez Descartes, le fondement a priori du système des connaissances n'est autre que l'ego théorique, doué d'une lumière donatrice d'intelligibilité, il se définit pour les Encyclopédistes comme prioritairement pratique, porteur d'une lumière révélatrice d'utilité: l'intérêt ne se mesure pas à l'aune de la connaissance, mais des besoins de l'homme qui, de là, en commandent l'intelligibilité. Apparaît donc déjà, sous la plume des Encyclopédistes, une première requalification de la notion cartésienne de lumière naturelle - requalification qui valide une thèse majeure de Horkheimer et Adorno: puisqu'au XVIII' siècle, la lumière de l'esprit devient essentiellement pratique, il est vrai qu'il appartient aux Lumières d'avoir conduit à son paroxysme la conception instrumentale de la raison. De là, deux questions. Premièrement, pourquoi une telle requalification ? Deuxièmement, la critique de la métaphore cartésienne de la lumière n'engage-t-elle que la substitution, à titre de fondement du système des connaissances, d'un sujet pratique à un sujet théorique, tous deux posés de façon a priori?

\section{La critique de la lumen cartésienne}

D'un point de vue conceptuel, le système encyclopédique semble se démarquer de l'arbre cartésien par la distinction qu'il opère entre entendement humain et intellect divin. Selon D'Alembert, la chaîne des sciences n'est pas celle que Dieu perçoit et qui correspond à la façon dont les êtres de la nature se lient les uns les autres, mais celle que l'homme se résout à construire parce qu'une telle perception divine lui est refusée ${ }^{16}$. L'esprit humain ne participe pas de «l'intelligence suprême », et c'est pourquoi

15. Le système encyclopédique peut sembler à première vue plus « totalitaire » que l'arbre cartésien, précisément parce qu'il inclut non seulement les sciences, mais aussi l'ensemble des arts et métiers, là où Descartes estimaient que ceux-ci étaient réfractaires à la systématisation. Voir René Descartes, op. cit., p. 2.

16. Jean Le Rond d'Alembert, Article «Éléments des sciences », Encyclopédie, t. v (1755), p. 491 a-b. 


\section{Labyrinthe, $n^{\circ} 34$}

il lui faut faire un système: si Dieu peut se contenter du système qu'il a créé - l'Univers lui-même - l'homme quant à lui se voit contraint, pour ne pas s'y perdre, d'ordonner cet Univers en un système à sa mesure ${ }^{17}$. Si, comme la présence de la métaphore solaire le donne à penser, d'Alembert se réfère alors, de façon critique, aux premières lignes des Regulae $e^{18}$, faut-il estimer que Descartes, de son côté, ne conçoit qu'une seule et unique chaîne, visible aussi bien par l'homme que par Dieu?

Descartes n'assimile pas lumière naturelle et lumière divine, et préserve la différence entre les sciences telles que Dieu les a créées et la chaîne que l'homme est en charge de constituer: si, d'après lui, la lumière du sujet n'éclaire pas les objets comme le fait la lumière divine, mais selon sa propre capacité à les éclairer, c'est précisément que cette dernière lui est inaccessible. Comme le système encyclopédique, l'arbre de la connaissance tel que Descartes le conçoit vient pallier l'impossibilité d'accéder au point de vue de Dieu. Aussi ces deux systèmes reposent-ils tous deux sur le même constat: l'entendement humain est essentiellement fini - ce qui sera loin d'être le cas de l'Encyclopédie des sciences philosophiques de Hegel, qui prétendra élever la lumière naturelle dont l'homme dispose jusqu'à l'Esprit absolu de Dieu ${ }^{19}$. Mais il n'empêche que Diderot et d'Alembert ne tirent pas de la finitude de l'esprit humain les mêmes conséquences que leur prédécesseur.

L'ordre méthodique qui, chez Descartes, guide la constitution du système repose donc sur la distinction entre lumière naturelle et lumière divine: si l'homme avait accès au point de vue de Dieu sur l'Univers, il ne lui serait pas nécessaire d'ordonner les sciences selon sa capacité à en éclairer les objets, puisqu'il pourrait les envisager dans leur ordre naturel, telles qu'elles ont été créées par Dieu. Cependant, l'ordre que l'homme s'attache ainsi à construire lui permet de retrouver celui que Dieu a institué, de telle sorte que finalement, la lumière dont il dispose suscite le même éclairage que la lumière divine: selon Descartes, l'ordre que le sujet impose aux objets à connaître peut être considéré comme leur enchaînement naturel dès lors que les effets que cet ordre permet de

17. Jean Le Rond d'Alembert, DP, op. cit., p. 108. André Charrak, Contingence et nécessité des lois de la nature, Paris, Vrin, 2006, p. 10.

17. André Charrak, ibid., p. 9.

18. Apparaît ainsi que la critique que Adorno et Horkheimer adressent au système de l'Aufklärung s'applique de façon bien plus heureuse au système de Hegel. Voir sur ce point l'article d'Arnault Skornicki. 


\section{Système des connaissances et système du monde}

prédire se produisent effectivement. $\mathrm{Si}$, chez Descartes, lumière humaine et lumière divine ne se recouvrent pas, elles finissent cependant par projeter sur les choses la même luminosité ${ }^{20}$. Aussi la façon dont les objets se lient les uns aux autres n'est-elle pas hors de portée de l'entendement humain : l'ordonnancement auquel celui-ci procède prétend bien atteindre, quoique de façon médiate, leur ordre naturel ${ }^{21}$.

Contre Descartes, les Encyclopédistes estiment que le caractère inaccessible de la lumière divine ne doit pas inciter l'homme à renoncer à la connaissance par les effets pour forger un système a priori. Car selon eux, rien ne garantira que l'ordre ainsi construit sera celui-là même que Dieu a originellement créé. Les bornes de l'entendement humain sont telles qu'il y a même toutes les chances, souligne Condillac ${ }^{22}$, qu'un tel système ne soit au contraire que pure rêverie. Loin de venir éclairer la nature, la lumière à laquelle les Regulae soumettent l'enchainement des objets produit des images auxquelles rien ne correspond dans la réalité: certes elle illumine, mais, au lieu de projeter sa lumière sur ce qui paraît obscur à l'esprit, suscite une clarté toute fictive ${ }^{23}$. Pour les Encyclopédistes, tout se passe comme si Descartes, en attribuant à l'homme le pouvoir de créer le système de la nature de manière a priori, avait subrepticement transféré à la lumière naturelle les caractéristiques de la lumière divine. Voilà sans doute la raison pour laquelle Diderot et d'Alembert ne conçoivent pas l'apriorité de la lumière du

\footnotetext{
19. Peut-être pourrait-on dire alors que lumière divine et lumière naturelle se distinguent en tant que lux , ou source de lumière, mais produisent au final la même lumen, ou luminosité. Voir la lettre de Descartes à Morin du 13 juillet 1638: « La lumière, c'est-à-dire lux, est un mouvement ou une action dans le corps lumineux, et elle tend à causer quelque mouvement dans les corps transparents, à savoir lumen. Donc lux est première que lumen » (Euvres, établies par AdAm et TANNERY en 11 tomes, Paris, 1897-1909, rééd. par les éditions du CNRS et Vrin 1964-1974; 1996, t. II, p. 205.Titre abrégé par la suite en A.T. suivi du tome en chiffres romains et de la page en chiffres arabes.)

20. Tout en lui étant redevable de l'idée selon laquelle le «paradigme du soleil » contribue, chez Descartes, à substituer à la division aristotélicienne des sciences une unification de celles-ci gouvernée par l'ego cogito, nous ne pouvons nous accorder tout à fait avec Jean-Luc Marion qui s'appuie dans $o p$. cit.,p. 77, sur la lettre de Descartes à Mersenne du 10 mai 1632 pour conclure ainsi à la permanence du hiatus entre l'ordre méthodique et l'ordre des choses, donc entre lumière naturelle et lumière divine. Il semble bien plutôt que Descartes, dans ce texte, souligne qu'une saine méthode autorise la saisie d'un ordre naturel primitivement caché aux regards.

21. Étienne Bonnot, abbé de Condillac, Traité des systèmes, Paris, Fayard, Corpus, 1991 [1749], p. 4.

22. De la sorte, lorsqu'Adorno et Horkheimer écrivent d'une façon générale que « le système doit être maintenu en harmonie avec la nature; de même que les faits sont pronostiqués à partir du système, ils doivent à leur tour le confirmer » (op. cit., p. 93), ils identifient de façon indue système cartésien et système encyclopédique.
} 


\section{Labyrinthe, $n^{\circ} 34$}

sujet comme celle de sa puissance de connaître, et l'envisagent au contraire comme celle de son sentiment véritablement primitif, à savoir le besoin. Non seulement l'homme n'a pas la faculté de retrouver médiatement la lumière divine, mais il ignore le désintéressement qui est celui de Dieu: il cherche à connaître pour améliorer ses conditions de vie, ce qui, bien entendu, n'est pas le cas de l'être suprême. Aucun ego théorique ne saurait donc figurer de façon a priori au fondement du système des sciences.

Loin s'en faut cependant que l'impossibilité d'embrasser l'Univers par la pensée débouche selon eux sur l'abandon de toute idée de systématisation. L'Encyclopédie devra seulement se garder de réitérer l'erreur de Descartes : renoncer à connaître le monde par ses effets sous prétexte que la majorité d'entre eux échappe à la lumière de l'esprit.

\section{La refonte de la lumière naturelle}

La manière d'éviter que la lumière naturelle ne produise de simples rêveries s'impose alors d'elle-même. Plutôt que d'ordonner les objets selon sa capacité à les éclairer, elle partira des seuls principes véritables, à savoir les faits, principes sur lesquels «sont fondés les vrais systèmes » ${ }^{24}$.

Si les Encyclopédistes entendent, comme Descartes, pallier la finitude de l'esprit humain, ils emploient pour ce faire une voie contraire à l'apriorisme cartésien: cette voie consiste à partir de faits connus, véritables remparts aux rêveries, et de chercher de là comment ils peuvent expliquer d'autres faits dont, jusqu'à présent, la liaison avec les connaissances disponibles n'avait pas été mise au jour. Ce que Condillac pense sous la catégorie de « fait » n'est donc pas un simple énoncé d'observation, du type « l'aimant attire le fer ». Le « fait» condillacien enveloppe l'explication de ce qui se donne à voir, au point que l'abbé en vient à qualifier de tel la raison même du phénomène ${ }^{25}$.

La perspective des Regulae est ainsi radicalement modifiée. Le sujet n'impose plus son ordre à ses objets, mais vise à reproduire l'ordre qui leur est propre, i.e. les rapports qui sont les leurs indépendamment de la lumière par laquelle il vient les éclairer. En cela, les Encyclopédistes

24. Étienne Bonnot, abbé de Condillac, op. cit., p. 5.

25. Op. cit., p. 4-5. Sur cette distinction entre « fait» et «phénomène », essentielle à la refondation empiriste du concept de système, voir ci-contre l'entretien avec André Charrak. 


\section{Système des connaissances et système du monde}

retrouvent une posture aristotélicienne, sans pour autant revenir au dispositif du Stagirite. Les objets des sciences ne se rangent pas sous des genres incommunicables, mais se lient insensiblement les uns les autres. Qu'il n'existe pas, dans l'Univers, de fait absolument premier ${ }^{26}$ rend ainsi caduque l'idée de hiérarchisation des étants. Le concept qui préside à l'ordonnancement des sciences n'est donc plus celui de subordination ou de subalternation, mais celui d'application ${ }^{27}$ : une science en précède une autre dans l'arbre de la connaissance lorsqu'elle doit s'y appliquer pour en assurer la pertinence et la fécondité. C'est ainsi que les mathématiques, par la nécessité qu'elles octroient aux énoncés de la physique, autorisent cette science à percer les secrets de la nature ${ }^{28}$.

En somme, les Encyclopédistes refusent le renversement sujet/objet opéré par l'auteur des Regulae et rétablissent la primauté de l'objet au nom même de ce qui, chez Descartes, motivait un tel renversement - à savoir la distinction lumière naturelle/lumière divine: puisque le point de vue de Dieu sur le monde nous est refusé, nous ne pouvons constituer l'ordonnancement des choses tel que lui-même l'a créé, de façon a priori, et sommes condamnés à les envisager selon l'ordre imparfait et lacunaire dans lequel elles nous apparaissent ${ }^{29}$.

Voici donc les sciences reliées entre elles selon que les objets de l'une constituent les principes de la suivante. Seulement, nous obtenons ainsi non pas une chaîne ininterrompue, ni encore moins un arbre, mais des séquences lacunaires ${ }^{30}$. L'état historique du développement des connaissances fait que la lumière naturelle n'a jusqu'à présent éclairé qu'une infime partie de l'Univers, et sa faible luminosité qu'elle n'éclairera jamais tout ce qui pourrait l'être ${ }^{31}$. La liaison des sciences reste imparfaite dans la mesure où les rapports de fondation ne sont pas tous élucidés : il reste ainsi une multitude de disciplines auxquelles les mathématiques n'ont

\footnotetext{
26. Denis Diderot, article « Encyclopédie », op. cit., p. 641 b.

27. André Charrak, op. cit., p. 10-11.

28. Jean Le Rond d'Alembert, op. cit., p. 94.

29. Pour Descartes au contraire, l'impossibilité où nous sommes d'accéder à la lux divine nous contraint seulement à passer par une voie détournée, définie par la méthode, et qui consiste à imiter le caractère créateur d'une telle lumière pour s'attacher ensuite à vérifier si nous avons produit ainsi sur les choses la même lumen.

30. Jean Le Rond d'Alembert, Essai sur les éléments de philosophie, chap. Iv, p. 25-26.

31. Jean Le Rond d'Alembert, article « Eléments des sciences », op. cit., p. 491 b.
} 
pas encore été appliquées, quoiqu'elles le pourraient tout à fait ${ }^{32}$. Et les sciences elles-mêmes, qui sont autant de systèmes particuliers ${ }^{33}$ constitutifs du système général des connaissances, présentent de nombreuses lacunes: les propriétés de leurs objets ne sont pas toutes connues, ni, parmi celles qui le sont, les liens qui les unissent.

Ainsi, l'idée que se font les Encyclopédistes de la lumière naturelle conditionne l'élaboration d'un système qui ne peut qu'être inachevé: parce que la lumière du sujet se contente de projeter son faisceau sur le monde, tout ce qu'elle ne parvient pas à éclairer reste dans l'ombre, que ce soit ce que le progrès des connaissances lui permettra d'illuminer un jour, ou ce qui lui échappera toujours en raison de sa faible intensité. Et loin que ce qui demeure ainsi dans les ténèbres fasse obstacle à la constitution du système, celui-ci ne pourrait voir le jour s'il ne comportait aucune lacune. Car il deviendrait alors pour l'esprit humain aussi invisible que le système-Univers dont il serait l'exact refle $^{34}$ - système-Univers dont l'invisibilité a précisément déterminé l'esprit à ordonnancer ses connaissances. En somme, la chaîne des sciences se doit d'être incomplète pour paraître à l'entendement sous la figure du système. A contrario Descartes, parce que la lumière qu'il attribue à l'entendement humain ordonne les objets selon sa capacité à les éclairer, conçoit un système d'où les lacunes sont par définition exclues. Dans la mesure où la lumière naturelle ne vient pas éclairer une chaîne qui lui préexisterait, mais créer de toutes pièces un nouvel enchaînement, la continuité du faisceau lumineux devient celle-là même du système des connaissances.

Mais si, dans l'Encyclopédie, les sciences s'enchaînent selon la nature de leurs objets, et s'il n'existe cependant pour les fonder aucun fait absolument premier, rien ne peut objectivement les unifier et garantir ainsi leur systématicité. Comment un tel ordonnancement pourrait-il dès

32. C'est le cas notamment de la logique, ou « art de penser », que Condillac considère comme une sorte de mathématique universelle. Voir La logique ou les premiers développements de l'art de penser, Paris, Vrin, [1780] 1981, p. 313.

33. Étienne Bonnot, abbé de Condillac, Traité des systèmes, op. cit., p. 1. Quatre acceptions du mot «système » sont donc en usage au XVIII ${ }^{\mathrm{e}}$ siècle: $1^{\circ}$ le système-Univers $2^{\circ}$ le système-encyclopédie, ou ordonnancement raisonné des connaissances humaines $3^{\circ}$ le sous-système, ou science particulière $4^{\circ}$ le système-doctrine, conçu comme « le tout et l'ensemble d'une théorie dont les différentes parties sont liées entre elles, se suivent et dépendent les unes des autres. [...] C'est en ce sens là que l'on dit [...] le système de Descartes, celui de Newton, etc. » (article «Système », Encyclopédie, op. cit., t. xv, p. 778 a).

34. Denis Diderot, Article « Encyclopédie », op. cit., p. 641 a. 


\section{Système des connaissances et système du monde}

lors s'offrir à nous comme un système, ou un ensemble de parties reliées à un unique fondement ?

C'est seulement par la liaison de toutes les sciences à l'entendement humain que l'aporie vient se résoudre. L'ordre des séquences demeure celui que leur imposent leurs objets, de sorte qu'il est assuré que les connaissances ainsi ordonnancées ne sont pas de pures visions et portent bien sur la nature des choses. Et les sciences trouvent cependant le fondement nécessaire à leur systématicité, gage de visibilité, et par là même de progrès. La place qu'occupe l'entendement dans le système encyclopédique tient donc du plus grand des paradoxes : c'est parce que l'objet décide de son ordre et rend vaine l'idée de principe objectif qu'il ne reste plus qu'au sujet, en dernière instance, à fonder le système des connaissances. Par conséquent, parce que la lumière naturelle, au XVIII ${ }^{\mathrm{e}}$ siècle, ne vient plus, comme chez Descartes, disposer les objets selon l'ordre qu'elle leur impose, mais les éclairer, autant que faire se peut, dans celui qui est naturellement le leur, le sujet qui réalise l'unification des sciences ne précède pas la systématisation au titre d'unité posée $a$ priori, mais se découvre fondement du système de manière $a$ posteriori. La lumière naturelle telle qu'elle est conçue par les Encyclopédistes ne se réduit donc pas à la lux révélatrice d'utilité signalée par Diderot. Plus précisément, si l'éclairage qu'elle dispense est bien foncièrement intéressé, il est solidairement source d'intelligibilité, au sens où ne peut être utile que ce qui est connu.

De labyrinthique qu'il était, l'ordonnancement des connaissances prend dès lors la forme d'un arbre, dont le tronc est l'entendement humain, les trois principales branches l'histoire, la philosophie et la poésie, et leurs ramifications « toutes les autres sciences ${ }^{35}$ ». L'entendement humain est en mesure de relier les différentes sciences, parce que les trois facultés qui le composent - mémoire, raison, imagination - donnent lieu à ces trois disciplines auxquelles il est possible de relier toutes les autres. Si l'homme est posé de façon a priori par Diderot comme fin pratique à laquelle doit se subordonner le savoir, le DP lui attribue clairement le statut de fondement théorique du système, et ce de façon strictement $a$

35. Selon l'expression de René Descartes, Lettre-préface à la traduction française des Principia Philosophiae, A.T. IX, p. 14. 


\section{Labyrinthe, $n^{\circ} 34$}

posteriori, une fois les connaissances disposées selon l'ordre que leurs objets leur imposent. De ce point de vue, le dispositif cartésien est totalement renversé.

\section{Conclusion}

Que le système encyclopédique, à l'instar du système cartésien, situe à son fondement l'entendement humain, traduit paradoxalement l'écart qui s'est progressivement creusé entre la théorie de la connaissance de l'âge classique et celle du siècle des Lumières. C'est ainsi la requalification du concept de « lumière naturelle » qui, avant toute chose, autorise le passage de l'une à l'autre de ces conceptions du système: une telle lumière, au milieu du XvIII ${ }^{\mathrm{e}}$ siècle, ne crée plus ce qu'elle vient éclairer, mais restaure l'ordre des connaissances qui, selon celui de leur genèse, ne répandait guère que des ténèbres, et, autant qu'il est en elle, éclaire le monde tel qu'il a été créé par Dieu. Aussi le véritable système, celui qui, loin d'être une pure rêverie, reflète la nature des choses, paraît-il ouvert et inachevé. Dès lors n'exprime-t-il pas le désir d'embrasser l'Univers par la pensée, mais la conscience de l'impossibilité d'accéder à un point de vue qui n'appartient qu'à Dieu. S'il appartient bien à l'Aufklärung de faire l'apologie du système ${ }^{36}$, il lui revient aussi d'être l'initiatrice de sa critique dans son versant dogmatique, et le lieu d'un « esprit systématique » tout à fait conscient des limites de la lumière naturelle. L'entreprise des Encyclopédistes atteste donc à elle seule que l'interdisciplinarité peut et doit s'établir en un système, i.e. au travers d'une recherche des principes qui marquent pour chaque science les ressorts de ses progrès.

36. «[...] l'Aufklärung est la philosophie qui identifie la vérité au système scientifique. », Max Horkheimer et Theodor Adorno, op. cit., p. 95. 
Système des connaissances et système du monde 